\title{
Estrategias de negociación en parejas violentas y no violentas en Arequipa
}

Negotiation Strategies in Violent and Not-Violent Couples from Arequipa

\author{
Paula Delgado Cuzzi \\ Universidad Católica San Pablo, Perú \\ Correspondencia: pdelgado@ucsp.edu.pe
}

Recibido: 15-12-2015

Aceptado: 10-02-2016

\begin{abstract}
Resumen
Esta investigación comparó las diferencias en el uso de estrategias de negociación en parejas donde ha existido violencia en el último año y aquellas en las que no. Se realizó un estudio por encuestas, en donde participaron 279 adultos de la ciudad de Arequipa, quienes en el momento de ser encuestados convivían con su pareja o estaban casados. La muestra fue obtenida de tres distritos de la ciudad y los participantes fueron contactados y entrevistados en sus hogares. Se utilizó la Escala de Tácticas de Conflicto Revisada para medir el uso de tácticas de negociación e incidentes de violencia perpetrados por ambos miembros de la pareja. Se encontraron diferencias significativas entre las parejas en las que se reportó y aquellas en las que no $(t=-2.48, p<.05$ en violencia perpetrada por el participante, $t=-2.57, p<.05$ en violencia perpetrada por la pareja del participante). Diferencias significativas también se obtuvieron en negociación cognitiva y emocional en ambos casos. Los participantes que reportaron violencia en su relación de pareja también reportaron mayor frecuencia de uso de tácticas de negociación.
\end{abstract}

Palabras clave: Estrategias, negociación, violencia intrafamiliar, pareja violenta.

\begin{abstract}
The present study compared the differences in use of negotiation strategies in couples in which physical violence has occurred in the last year and couples in which it has not occurred. A survey research study was conducted, 279 adults from Arequipa city participated, who were married or cohabiting with a partner at the time they were surveyed. The sample was obtained from 3 districts of the city and the participants
\end{abstract}


were contacted and surveyed at their homes. The Conflict Tactics Scale Revised was used to assess the use of negotiation tactics and physical violence incidents perpetrated by both partners. Significant differences between the couples in which violence was reported and the ones in which violence was not reported were found $(t=-2.48, p<.05$ for violence perpetrated by the participant, $\mathrm{t}=-2.57, \mathrm{p}<.05$ for violence perpetrated by the participant's partner). Significant differences were also found for cognitive and emotional negotiation in both cases. Participants that reported violence in their couple relationship also reported a higher frequency of use of negotiation tactics.

Key words: Strategies, negotiation, inside-violent family, violent couple.

\section{Introducción}

Los conflictos en las relaciones de pareja son inevitables, y la forma en la que son manejados afecta el nivel de satisfacción que sienten los miembros por su relación de pareja y la estabilidad de esta (Bertoni \& Bodenmann, 2010; Fitzpatrick, 1988; Gottman, 1993). Los conflictos también pueden escalar hasta la violencia. Por ejemplo, Johnson $(1995,2011)$ describe tres tipos de violencia en las relaciones. El primero representa lo que normalmente se conoce como violencia contra la mujer, lo cual incluye intentos de controlar a la otra persona, así como un uso sistemático de agresión. El segundo tipo describe las respuestas violentas de alguien que sufre el primer tipo. Sin embargo, el tercer tipo planteado por Johnson (violencia situacional de pareja) hace referencia al conflicto que se sale de control; este empieza en formas leves, y posteriormente puede escalar a formas más severas. Este tipo, usualmente visto como menos importante, incluye la mayoría de casos de violencia en las parejas y puede escalar a formas severas y potencialmente letales (Delgado, 2015; Feld \& Straus, 1979; Johnson, 2011; Johnson \& Leone, 2005). Además, es el tipo de violencia más comúnmente reportado en encuestas en la población general, ya que se cree que víctimas del primer tipo, llamado terrorismo íntimo, son más propensas a tener miedo de reportar la situación (Johnson, 1995, 2006, 2011).

En Perú, además, es de vital importancia estudiar la violencia en las relaciones de pareja y los factores asociados a esta, debido a las altas prevalencias en las parejas. Diferentes estudios de investigación en el país sobre la violencia contra la mujer en la pareja han reportado prevalencias entre $30.9 \%$ y $38.9 \%$ (Flake, 2005; Gonzales \& Gavilano, 1999). Sin embargo, en el 2006, la Organización Mundial de la Salud reportó prevalencias más altas, de $48.6 \%$ para Lima y $61.0 \%$ para provincias (Garcia-Moreno, Jansen, Ellberg, Heise \& Watts, 2006). Asimismo, en Perú, a pesar de ser mucho menos estudiado, para los hombres, el riesgo de sufrir violencia en la relación de pareja es bastante similar al de las mujeres (Fiestas, Rojas, Gushiken \& Gozzer, 2012). Por otra parte, en una investigación en Arequipa, cerca de tres cuartos de los participantes reportaron violencia en su relación de pareja y la gran 
mayoría de ellos reportó violencia bidireccional, la cual es más probable que escale a niveles más severos y a un mayor número de lesiones (Delgado, 2015). Agregado a esto, pocas investigaciones han desarrollado el tema de los conflictos en la pareja y factores relacionados en poblaciones que no sean europeas o norteamericanas, lo cual es particularmente importante en Perú, por la ya mencionada alta prevalencia de violencia en las relaciones de pareja y las significativas diferencias culturales con las poblaciones donde este fenómeno ha sido estudiado.

\section{Negociación}

En la Escala de Tácticas de Conflicto (Conflict Tactics Scale Revised - CTS2), la negociación es medida como aquellas acciones tomadas para resolver un conflicto a través de discusiones y la comunicación de afecto positivo hacia la pareja durante esa situación (Straus, Hamby \& Warren, 2003). Esta, a su vez, hace una diferenciación entre estrategias cognitivas y emocionales de negociación. En cuanto a las estrategias cognitivas, hacen referencia a los intentos de solucionar mediante la conversación y propuesta de soluciones conjuntas. Y complementario a esto, las estrategias emocionales aluden a la expresión de respeto y afecto hacia la otra persona. La capacidad de negociación en la pareja es también necesaria para una buena percepción de la relación. Scanzoni (1982) refiere que el conflicto puede ser positivo en las relaciones de pareja, y la oportunidad de negociar aquellos aspectos que lo generan inicialmente y llegar a un consenso pueden hacer que se perciba la relación con un mayor grado de reciprocidad y solidaridad.

\section{Conflicto, negociación y violencia}

Gottman (1993) ha propuesto 5 tipos de pareja, de acuerdo a sus aproximaciones a los conflictos, los cuales podían favorecer o no a la estabilidad de la pareja. Él, además, llegó a la conclusión de que evitar o llegar a altos niveles de conflicto no es necesariamente negativo. La diferencia entre los estilos estables que propone (volátiles, validadores y evitadores) y los estilos inestables (hostiles y hostiles/ desconectados) era que los estables mantenían por lo menos un ratio de cinco interacciones positivas (como presentación positiva de los temas a tratar, gestos de asentimiento, humor, o escucha positiva) por cada interacción negativa (como evasivas, crítica, desprecio o actitud o respuestas defensivas) durante los conflictos. Similar a esta propuesta, Bertoni y Bodenmann (2010) encontraron que la insatisfacción de pareja estaba relacionada con niveles altos de ofensas y evitación del conflicto. Asimismo, esta insatisfacción estaba relacionada también a la violencia física.

Hurtado, Ciscar \& Rubio (2004) proponen que el conflicto crónico en las parejas es una muestra de falta de habilidades sociales necesarias para solucionarlo. Esto, 
a su vez, aumentaba la probabilidad de conductas de violencia en la relación de pareja. Con respecto a esto, Babcock, Waltz, Jacobson y Gottman (1993) encontraron que los déficit en las habilidades de comunicación en los miembros de la pareja incrementaban el riesgo de violencia física, posiblemente por la falta de recursos para resolver el conflicto o usar estrategias verbales para suavizarlo. Moral, López, Díaz-Loving y Cienfuegos (2011) también encontraron que la manifestación de afecto en mujeres durante el conflicto disminuían la violencia.

El presente estudio tiene un diseño de investigación por encuestas (Heppner, Wampold \& Kivlighan, 20o8), en donde se examinó la presencia de violencia física, perpetrada tanto por el participante como por su pareja, y las tácticas de negociación utilizadas por el encuestado. Así mismo, la investigación también buscó identificar cómo estas están relacionadas.

\section{Método}

\section{Participantes}

La muestra de la presente investigación fue obtenida en la comunidad, en donde los participantes fueron contactados en sus casas. Tres distritos de Arequipa fueron seleccionados: Paucarpata, 36.2 \%, Cerro Colorado, 43.0 \%, y Hunter, 20.8 \%. La muestra dentro de estos distritos fue obtenida aleatoriamente estratificada por sexo.

La muestra inicial estuvo compuesta por 300 adultos viviendo en Arequipa, sin embargo la muestra se redujo a 279 , ya que el número restante de participantes reportaron ser solteros en el momento de la entrevista o no completaron todas las preguntas de la escala. Un número similar de hombres y mujeres participaron $(49.1 \%$ y $50.9 \%$ respectivamente). Las edades de estos estaban entre 18 y 65 años. La mayoría de ellos estaban casados al momento de la encuesta (84.6\%). El número restante de encuestados vivía con su pareja (15.4\%).

Por aspectos de ética y para reducir el riesgo de posibles represalias, solo un miembro de la pareja fue entrevistado sobre la presencia de violencia en el último año. El 71.o \% de los participantes reportó, por lo menos, un incidente de violencia física perpetrada hacia su pareja y el $63.8 \%$ reportó, por lo menos, un incidente de violencia perpetrado por su pareja hacia este (véase Tabla 1 para información sobre características sociodemográficas). 
Tabla 1.

Características sociodemográficas de los participantes $(n=279)$

\begin{tabular}{|l|c|c|}
\hline \multicolumn{1}{|c|}{ Variable } & $\mathrm{n}$ & $\%$ \\
\hline Distrito & & \\
Paucarpata & 101 & 36.2 \\
Cerro Colorado & 120 & 43.0 \\
Hunter & 58 & 20.8 \\
\hline Sexo & & \\
Femenino & 142 & 50.9 \\
Masculino & 137 & 49.1 \\
\hline Estado Civil & & \\
Casado & 236 & 84.6 \\
Conviviente & 43 & 15.4 \\
\hline Edad & & \\
$18-24$ & 34 & 12.2 \\
$25-34$ & 90 & 32.3 \\
$35-44$ & 62 & 22.2 \\
$45-54$ & 59 & 21.1 \\
$55+$ & 34 & 12.2 \\
\hline Violencia perpetrada por el participante & & \\
No & 198 & 29.0 \\
Sí & 178 & 63.8 \\
\hline Violencia perpetrada por la pareja del participante & & \\
No & & \\
Sí & & \\
\hline
\end{tabular}

\section{Instrumentos}

El uso de estrategias de negociación y la presencia de violencia física en la pareja fueron evaluados usando la Escala de Tácticas de Conflicto Revisada (CTS2) (Straus, Hamby, Boney-McCoy \& Sugarman, 1996). La escala está compuesta por 78 preguntas con ocho opciones de respuesta que van desde "nunca ha ocurrido" a "más de 20 veces el año pasado”. La CTS2 tiene 5 subescalas: agresión física, agresión psicológica, coerción sexual, lesiones y negociación. Para la presente investigación solo se utilizaron las subescalas de agresión física y negociación. Además, la escala de negociación tiene dos subescalas, una emocional y otra cognitiva. 
Para la aplicación de la escala en Arequipa, la traducción fue revisada y algunos de los términos fueron adaptados al lenguaje de la población (Delgado, 2015). El uso de la escala y las modificaciones en la traducción fueron aprobados por Western Psychological Services (WPS). La confiabilidad interna de la prueba fue obtenida usando la misma muestra por Delgado (2015), en cuanto a las subescalas de Perpetración de violencia física (.822) y de Victimización de violencia física (.837). Con respecto a la escala de Negociación, la confiabilidad interna para la subescala del Uso de negociación del participante en la investigación fue de .759 , y del Uso de estrategias de negociación de su pareja fue de .782 , obtenidas en base a la muestra de la presente investigación.

\section{Procedimiento}

La aplicación de las encuestas fue aprobada por el Comité de Revisión Institucional (Institutional Review Board) de Saint Cloud State University. Los encuestadores y otras personas involucradas en el proceso de encuesta de los participantes y recolección de la información fueron capacitados sobre la finalidad del proyecto, información básica sobre violencia en las parejas, recomendaciones y procedimientos de seguridad. El consentimiento informado de los participantes fue obtenido oralmente, una vez que el encuestador y el entrevistador se encontraban a solas. Información de contacto del investigador principal e información de contacto de recursos para casos de violencia contra la mujer o terapia de pareja fueron ofrecidos al participante. La encuesta fue llenada por el participante en papel mientras el encuestador se encontraba cerca, en caso el encuestado tuviera preguntas sobre la escala y por motivos de seguridad. Las consideraciones y recomendaciones éticas propuestas por la Organización Mundial de la Salud se siguieron durante la investigación (Watts, Heise, Ellsberg \& García, 2001).

\section{Análisis estadístico}

Las diferencias en el uso de estrategias de negociación en general, de negociación cognitiva y emocional usados por el participante entre parejas en las que este reportó, por lo menos, un incidente de violencia hacia su pareja en el último año y aquellas en las que no hubo ningún incidente fueron analizados usando la prueba t de Student para muestras independientes. Un análisis similar se hizo para evaluar las diferencias entre parejas donde el participante recibió, al menos, un acto de violencia física de su pareja y aquellos que no reportaron ningún incidente de violencia hacia ellos. El análisis de los resultados se realizaron utilizando SPSS 20.0 (IBM Corp, 2011). 


\section{Resultados}

Los resultados muestran diferencias en el uso de negociación entre parejas en donde hubo en el último año, como mínimo, un incidente de violencia perpetrada por los participantes y aquellas en las que no hubo ninguno. En la parejas donde el participante fue físicamente agresivo, las medias de uso de negociación $(\mathrm{M}=3 \cdot 31, \mathrm{DE}=1.36)$, negociación cognitiva $(\mathrm{M}=3.42, \mathrm{DE}=1.54)$ y negociación emocional $(\mathrm{M}=3.19, \mathrm{DE}=$ 1.50) fueron mayores a las medias de las mismas en donde el participante no reportó incidentes de violencia $(\mathrm{M}=\mathbf{2 . 8 4}, \mathrm{DE}=\mathbf{1 . 6 0})$ para negociación $(\mathrm{M}=\mathbf{2 . 9 3}, \mathrm{DE}=\mathbf{1 . 8 2})$, para negociación cognitiva $(\mathrm{M}=\mathbf{2 . 7 3}, \mathrm{DE}=1.69)$ y para negociación emocional. Además, estas diferencias fueron significativas estadísticamente $(\mathrm{t}=\mathbf{- 2 . 4 8}, \mathrm{p}<.05$ para negociación, $\mathrm{t}=\mathbf{- 2 . 0 8}, \mathrm{p}<.05$ para negociación cognitiva $\mathrm{y} \mathrm{t}=\mathbf{- 2 . 2 4}, \mathrm{p}<.05$ para negociación emocional) (véase tabla 2).

\section{Tabla 2.}

Resultados de t de Student y estadísticas descriptivas de negociación, negociación cognitiva y negociación emocional en violencia perpetrada por el participante

\begin{tabular}{|c|c|c|c|c|c|c|c|c|c|}
\hline & \multicolumn{6}{|c|}{ Violencia en el último año } & \multirow{2}{*}{\multicolumn{3}{|c|}{$\begin{array}{c}95 \% \text { IC para diferencia } \\
\text { de medias }\end{array}$}} \\
\hline & \multicolumn{3}{|c|}{ No } & \multicolumn{3}{|c|}{ Sí } & & & \\
\hline & M & $\mathrm{DE}$ & $\mathrm{n}$ & M & $\mathrm{DE}$ & $\mathrm{n}$ & Rango & $\mathrm{t}$ & df \\
\hline Negociación & 2.84 & 1.60 & 81 & 3.31 & 1.36 & 198 & $-.87,-.10$ & $-2.48^{*}$ & 277 \\
\hline Negociación cognitiva & 2.93 & 1.82 & 81 & 3.42 & 1.54 & 198 & $-.93,-.02$ & $-2.08^{*}$ & 129.75 \\
\hline Negociación emocional & 2.73 & 1.69 & 81 & 3.19 & 1.50 & 198 & $-.86,-.06$ & $-2.24 *$ & 277 \\
\hline
\end{tabular}

Nota: $t$ de Student para varianzas desiguales usado en el caso de negociación cognitiva ${ }^{*} \mathrm{p}<.05$

Resultados similares fueron obtenidos al comparar las medias del uso de negociación, negociación cognitiva y negociación emocional usada por el encuestado en parejas donde el participante fue el receptor de, al menos, un acto de violencia física en el último año y parejas en donde no se reportó ningún acto de este tipo. La media del uso de negociación en parejas donde hubo algún incidente de violencia contra el participante $(\mathrm{M}=3 \cdot 34$, DE $=1.32$ ) fue mayor a la media de parejas donde no se reportó este tipo de violencia $(\mathrm{M}=$ 2.86, $\mathrm{DE}=1.61)$. Esto es similar en la negociación cognitiva $(\mathrm{M}=3.47, \mathrm{DE}=1.53$, para parejas donde hubo violencia física, y $\mathrm{M}=\mathbf{2 . 9 4}, \mathrm{DE}=\mathbf{1 . 7 7}$, en parejas donde no la hubo) y en la negociación emocional $(\mathrm{M}=3.22, \mathrm{DE}=1.50$, para parejas donde hubo violencia física, y $\mathrm{M}=\mathbf{2 . 7 8}, \mathrm{DE}=1.69$, para parejas donde no la hubo). Estas diferencias fueron estadísticamente significativas $(\mathrm{t}=\mathbf{- 2 . 5 7}, \mathrm{p}<.05$ para negociación, $\mathrm{t}=-\mathbf{2} .51, \mathrm{p}<.05$ para negociación cognitiva, $\mathrm{t}=\mathbf{- 2 . 1 5}, \mathrm{p}<.05$ para negociación emocional) (véase Tabla 3). 


\section{Tabla 3.}

Resultados de t de Student y estadísticas descriptivas de negociación, negociación cognitiva y negociación emocional en violencia perpetrada por la pareja del participante

\begin{tabular}{|c|c|c|c|c|c|c|c|c|c|}
\hline & \multicolumn{6}{|c|}{ Violencia en el último año } & \multirow{2}{*}{\multicolumn{3}{|c|}{$\begin{array}{c}95 \% \text { IC para diferencia } \\
\text { de medias }\end{array}$}} \\
\hline & \multicolumn{3}{|c|}{ No } & \multicolumn{3}{|c|}{ Sí } & & & \\
\hline & M & $\mathrm{DE}$ & $\mathrm{n}$ & M & $\mathrm{DE}$ & $\mathrm{n}$ & Rango & $\mathrm{t}$ & df \\
\hline Negociación & 2.86 & 1.61 & 101 & 3.34 & 1.32 & 178 & $-.86,-.11$ & $-2.57^{*}$ & 176.33 \\
\hline Negociación cognitiva & 2.94 & 1.77 & 101 & 3.47 & 1.53 & 178 & $-.94,-.11$ & $-2.51 *$ & 183.97 \\
\hline Negociación emocional & 2.78 & 1.69 & 101 & 3.22 & 1.50 & 178 & $-.84,-.04$ & $-2.15^{*}$ & 176.40 \\
\hline
\end{tabular}

Nota: t de Student para varianzas desiguales usado en todos los casos

${ }^{*} \mathrm{p}<.05$

\section{Discusión}

La revisión de la literatura sugiere que las parejas que usan con mayor frecuencia tácticas de negociación verbal o afectiva deberían tener menos probabilidades de llegar a la violencia física en los conflictos (Babcock et al., 1993; Hurtado et al., 2004; Moral et al., 2011). Sin embargo, los resultados de la presente investigación muestran que los participantes que se encuentran en una relación donde ha habido, por lo menos, un acto de violencia física en el último año, hacia el participante o su pareja, usan con mayor frecuencia tácticas de negociación.

Una hipótesis para explicar los resultados de la presente investigación es que los participantes que se encuentran en relaciones violentas utilicen estrategias de negociación como consecuencia de la ocurrencia de actos violentos previos en la pareja, o como una táctica para evitar la posible recurrencia de los mismos. No obstante, los resultados de la presente investigación no clarifican si es que el uso de estas estrategias es mayor en las parejas luego de los incidentes de violencia. Esto es una de las limitaciones de este estudio, ya que al haber sido recolectada la información solamente en un periodo de tiempo no es posible identificar cuál de las variables ocurrió primero y si alguna de ellas fue consecuencia de la otra. Esto también sugeriría que, al intentar reducir la probabilidad de violencia física utilizando estas estrategias, la violencia o la intensidad del conflicto podrían verse reducidos, sin embargo, esto tampoco es comprobable en este estudio y debería ser considerado en investigaciones posteriores sobre el tema.

Esta hipótesis podría estar relacionada, además, por lo propuesto por Gottman (1993). En su teoría sobre los conflictos en las relaciones, él propone que las relaciones estables mantienen un ratio de cinco interacciones positivas por cada interacción negativa en conflictos. A pesar de que en su modelo, Gottman no toma en consideración la 
violencia física, sino únicamente muestras verbales o no verbales de crítica, actitud defensiva, evasivas o desprecio, algunas de ellas rozando lo considerado como violencia psicológica, esto podría sugerir que en las parejas donde existe violencia física, especialmente en formas leves, como lo es en la mayoría de la presente muestra (revisar Delgado [2015] para medias de incidentes de violencia leve y severa), las estrategias de negociación "positivas" son un mecanismo para mantener la estabilidad de dichas relaciones. Esto está además respaldado por el hecho de que, aunque en la mayoría de relaciones se reportaron incidentes de violencia, todos los participantes aún mantenían la relación de pareja. Pero, para una mejor comprensión de este fenómeno, se deberían desarrollar estudios longitudinales o incluir en la muestra relaciones en donde recientemente ha habido divorcio.

Como idea complementaria a la hipótesis anterior, se debería estudiar la cultura y sociedad donde la presente investigación fue realizada, ya que las altas prevalencias de violencia, la alta probabilidad de reciprocidad (Delgado, 2015; Flake, 2005; García-Moreno, et al., 2006; Gonzales \& Gavilano, 1999) y su relación con otras tácticas de solución de conflictos podrían ser algunos indicadores de una aceptación general de la violencia a nivel cultural. Esta aceptación en las relaciones de pareja y las normas culturales de lo que es esperable en estas influyen en la cantidad de violencia en las mismas (Carlson, 1984). Además, esta podría estar siendo percibida como una estrategia válida para afrontar conflictos en las relaciones de pareja, de forma que su uso es común junto con otras estrategias de negociación.

A pesar del aporte de la presente investigación para expandir el conocimiento de variables asociadas con el uso de violencia física en las relaciones, como lo son otras estrategias de negociación, este estudio tiene ciertas limitaciones que deberían considerarse y que pueden proponer sugerencias para investigaciones posteriores. Primero, la información fue recolectada a través de encuestas en donde los participantes reportan los casos de violencia, así como el uso de otras estrategias de negociación. En casos de violencia, la información obtenida por auto-reporte puede verse afectada por intentos de dar una impresión más positiva de uno (Archer, 1999). Esto, además, podría afectar los reportes de uso de estrategias de negociación cognitiva o emocional, como un intento de justificar o minimizar el uso de violencia física, algo así como «intentamos usar otras estrategias de solución de conflictos, pero no dieron resultado y tuvimos que usar la violencia física». Por lo tanto, la investigación futura podría incluir otras formas de evaluar el uso de estrategias de negociación o violencia. Segundo, otros tipos de violencia no fueron evaluados en este estudio. Esto favorecería a identificar el uso de negociación en diferentes tipos de violencia, como en violencia situacional en comparación con terrorismo íntimo, o incluyendo psicológica y sexual. 


\section{Referencias}

Archer, J. (1999). Assessment of the reliability of the Conflict Tactics Scales: A meta-analytic review. Journal of Interpersonal Violence, 14(12), 1263-1298. doi: 10.1177/088626099014012003

Babcock, J. C., Waltz, J., Jacobson, N. S., \& Gottman, J. M. (1993). Power and violence: The relation between communication patterns, power discrepancies, and domestic violence. Journal of Consulting and Clinical Psychology, 61(1), 40-50.

Bertoni, A., \& Bodenmann, G. (2010). Satisfied and dissatisfied couples: Positive and negative dimensions, conflict styles, and relationships with family of origin. European Psychologist, 15(3), 175-184. doi: 10.1027/1016-9040/aoooo15

Carlson, B. F. (1984). Causes and maintenance of domestic violence: An ecological analysis. Social Service Review, 58(4), 569-587.

Delgado, P. (2015). Reciprocal Violence and Injuries in Couples in Arequipa, Peru (Tesis de maestría). Recuperado de http://repository.stcloudstate.edu/cpcf_etds/4

Feld, S. L., \& Straus, M. A. (1989). Escalation and desistance of wife assault in Marriage. Criminology, 27(1), 141-161. doi 10.1111/j.1745-9125.1989.tboo866

Fiestas, F., Rojas, R., Gushiken, A., \& Gozzer, E. (2012). ¿Quién es la víctima y quién el agresor en la violencia física entre parejas? Estudio epidemiológico en siete ciudades del Perú. Revista Peruana de Medicina Experimental y Salud Pública, 29(1), 44-52.

Fitzpatrick, M. A. (1988). Negotiation, problem solving and conflict in various types of marriages. En P. Noller y Fitzpatrick, M. A. (Eds.), Perspectives on marital interaction: Monographs in Social Psychology of Languaje 1 (pp. 245 - 270) . Philadelphia, PA, Estados Unidos: Multilingual Matters Ltd.

Flake, D. F. (2005). Individual, family and community risk markers for domestic violence in Peru. Violence Against Women, 11(3), 353-373. doi: 10.1177/1077801204272129.

Garcia-Moreno, C., Jansen, H. A. F. M., Ellberg, M., Heise, L., \& Watts, C. H. (2006). Prevalence of intimate partner violence: Findings from the WHO multi-country study on women's health and domestic violence. Lancet, 386, 1260-1269.

Gonzales, E., \& Gavilano, P. (1999). Does poverty cause domestic violence? Some answers from Lima. En A. R. Morrison \& M. L. Biehl (Eds.). Too Close to Home. Domestic Violence in the Americas (35-49). Washington D. C.: Inter-American Development Bank.

Gottman, J. M. (1993). The roles of conflict engagement, escalation, and avoidance in marital interaction: A longitudinal view of five types of couples. Journal of Consulting and Clinical Psychology, 61(1), 6-15.

Heppner, P. P., Wampold, B. E., \& Kivlighan, D. M. (2008). Research Design in Counseling (3. ${ }^{\text {a }}$ ed.). Belmont, CA: Thomson Brooks/Cole. 
Hurtado, F., Ciscar, C., \& Rubio, M. (2004). El conflicto de pareja como variable asociada a la violencia de género contra la mujer: Consecuencias sobre la salud sexual y mental. Revista de Psicopatología y Psicología Clínica, 9(1), 49-64.

IBM Corp (2011). IBM SPSS Statistics for Windows, Version 20.o. Armonk, NY: IBM Corp.

Johnson , M. P. (1995). Patriarchal terrorism and common couple violence: Two forms of violence against women. Journal of Marriage and Family, 57(2), 283-294.

Johnson, M. P. (2006). Conflict and control: Gender symmetry and asymmetry in domestic violence. Violence Against Women, 12(11), 1003-1018. doi 10.1177/1077801206293328

Johnson, M. P. (2011). Gender and types of intimate partner violence: A response to antifeminist literature review. Aggression and Violent Behavior, 16, 289-296. doi. 10.1016/j.avb.2011.04.006

Johnson, M. P., \& Leone, J. M. (2005). The differential effects of intimate terrorism and situational couple violence: Findings from the National Violence against Women Survey. Journal of Family Issues, 26(3), 322-349. doi. 10.1177/0192513x04270345

Moral, J., López, F., Díaz-Loving, R., \& Cienfuegos, Y. I. (2011). Diferencias de género en afrontamiento y violencia en la pareja. Revista CES Psicología, 4(2), 29-46.

Scanzoni, J. (1982). Marital conflict as a positive force. En J. Scanzoni (Ed.), Sexual Bargaining: Power politics in the American Marriage (2. ${ }^{a}$ ed.) (pp. 61 - 102). Chicago, IL, Estados Unidos: The University of Chicago Press.

Straus, M. A., Hamby, S. L., Boney-McCoy, S., \& Sugarman, D. B. (1996). The Revised Conflict Tactics Scale (CTS2): Development and preliminary psychometric data. Journal of Family Issues, 17(3), 283-316. doi. 10.1177/019251396017003001

Straus, M. A., Hamby, S. L., \& Warren, W. L. (2003). The conflict tactics scales handbook. Los Angeles, CA, Estados Unidos: Western Psychological Services.

Watts, C., Heise, L., Ellsberg, M,. \& García, C. (2001). Putting Women First: Ethical and Safety Recommendations for Research on Domestic Violence Against Women. Geneva: World Health Organization. Recuperado de http://whqlibdoc.who.int/ hq/20o1/WHO_FCH_GWH_o1.1.pdf 